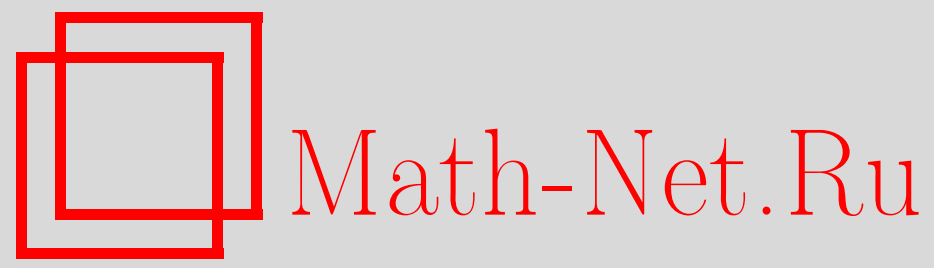

И. А. Ташков, Улучшение неравенства Массарта для статистики Смирнова, Вестник ТвГУ. Серия: Прикладная математика, 2018, выпуск 1, 5-20

DOI: https://doi.org/10.26456/vtpmk489

Использование Общероссийского математического портала Math-Net.Ru подразумевает, что вы прочитали и согласны с пользовательским соглашением

http://www . mathnet.ru/rus/agreement

Параметры загрузки:

IP : 54.80 .73 .141

26 апреля 2023 г., 16:34:31 


\section{ТЕОРИЯ ВЕРОЯТНОСТЕЙ И МАТЕМАТИЧЕСКАЯ СТАТИСТИКА}

УДК 519.2

\section{УЛУЧШЕНИЕ НЕРАВЕНСТВА МАССАРТА ДЛЯ СТАТИСТИКИ СМИРНОВА}

Ташков И.А.

МГУ им. М.В. Ломоносова, г. Москва

Поступила в редакцию 10.12.2017, после переработки 25.12.201\%.

Обозначим через $F_{n}$ эмпирическую функцию распределения по выборке из n независимых одинаково распределенных случайных величин с функцией распределения F. В данной работе доказано неравенство

$$
\mathbb{P}\left\{\sqrt{n} \sup _{|x|<\infty}\left(F_{n}(x)-F(x)\right)>\lambda\right\} \leq \exp \left\{-2 \lambda^{2}-\lambda^{4} / 36 n\right\}
$$

для $n \geq 39, \min \left\{\gamma n^{-1 / 6}, \sqrt{\ln 2 / 2}\right\} \leq \lambda \leq \sqrt{n} / 2, \gamma=1.0841$. Кроме того, доказано для тех же $n$ и $\lambda \leq \sqrt{n} / 2$, что

$$
\mathbb{P}\left\{\sqrt{n} \sup _{|x|<\infty}\left(F_{n}(x)-F(x)\right)>\lambda\right\} \leq 2 \exp ^{(\ln 2)^{2} /(144 n)} \exp \left\{-2 \lambda^{2}-\lambda^{4} / 36 n\right\} .
$$

Для частных случаев $n=2,3,4$ доказана более сильная оценка

$$
\mathbb{P}\left\{\sqrt{n} \sup _{|x|<\infty}\left(F_{n}(x)-F(x)\right)>\lambda\right\} \leq \exp \left\{-2 \lambda^{2}-4 \lambda^{4} / 9 n\right\} .
$$

Ключевые слова: распределение статистики Смирнова, экспоненциальные неравенства.

Вестник ТвГУ. Серия: Прикладная математика. 2018. № 1. С. 5-20. https://doi.org/10.26456/vtpmk489

\section{Введение}

Пусть независимые одинаково распределенные случайные величины $X_{n}, n \in \mathbb{N}=\{1,2, \ldots\}$, определены на вероятностном пространстве $(\Omega, \mathcal{F}, \mathbb{P})$. Предположим, что их функция распределения $F(x)=\mathbb{P}\left\{X_{1} \leq x\right\}, x \in \mathbb{R}=(-\infty, \infty)$ непрерывна. Напомним определение эмпирической функции распределения

$$
F_{n}(x)=\frac{1}{n} \sum_{k=1}^{n} \mathbb{I}_{\left\{X_{k} \leq x\right\}}, x \in \mathbb{R}=(-\infty, \infty),
$$


где $\mathbb{I}_{A}$ - индикаторная функция множества $A$. Смирнов [1] показал, что распределение статистики $D_{n}^{+}=\sqrt{n} \sup _{x \in \mathbb{R}}\left(F_{n}(x)-F(x)\right)$ имеет вид

$$
\begin{aligned}
& \mathbb{P}\left\{D_{n}^{+}>\lambda\right\}=1, \lambda<0 ; \mathbb{P}\left\{D_{n}^{+}>\lambda\right\}=0, \lambda \geq \sqrt{n}, \\
& \mathbb{P}\left\{D_{n}^{+}>\lambda\right\}=q_{\lambda, n}(0)+\lambda \sqrt{n} \sum_{k=[\lambda \sqrt{n}]+1}^{n-1} q_{\lambda, n}(k), 0 \leq \lambda<\sqrt{n}, \\
& q_{\lambda, n}(0)=\left(1-\frac{\lambda}{\sqrt{n}}\right)^{n}, q_{\lambda, n}(k)=\frac{1}{n^{n}}\left(\begin{array}{l}
n \\
k
\end{array}\right)(k-\lambda \sqrt{n})^{k}(n-k+\lambda \sqrt{n})^{n-k-1} .
\end{aligned}
$$

Символ $[\lambda \sqrt{n}]$ обозначает целую часть числа $\lambda \sqrt{n}$.

В статье Дворецкого, Кифера и Волфовица [2] доказано неравенство

$$
\mathbb{P}\left\{D_{n}^{+} \geq \lambda\right\} \leq c e^{-2 \lambda^{2}}
$$

с неопределенной постоянной $c$. Краткую историю вычислений данной константы можно найти в статье Массарта [3]. Там же приведено доказательство неравенства

$$
\mathbb{P}\left\{D_{n}^{+} \geq \lambda\right\} \leq e^{-2 \lambda^{2}} \text { для } \lambda \geq \min \left\{\gamma n^{-1 / 6}, \sqrt{\ln 2 / 2}\right\},
$$

где $\gamma=1.0841$. В данной статье доказано, что

$$
\mathbb{P}\left\{D_{n}^{+} \geq \lambda\right\} \leq \exp \left\{-2 \lambda^{2}-\frac{1}{36 n} \lambda^{4}\right\}, \min \left\{\gamma n^{-1 / 6}, \sqrt{\ln 2 / 2}\right\} \leq \lambda \leq \sqrt{n} / 2, n \geq 39 .
$$

Заметим, что $e^{-2 \lambda^{2}-\lambda^{4} / 36 n}>2^{-1} e^{-(\ln 2)^{2} /(144 n)}$ для $0 \leq \lambda \leq \sqrt{\ln 2 / 2}$. Отсюда и из (2) следует неравенство

$$
\mathbb{P}\left\{D_{n}^{+}>\lambda\right\} \leq 1 \leq 2 e^{(\ln 2)^{2} /(144 n)} e^{-2 \lambda^{2}-\lambda^{4} / 36 n}, 0 \leq \lambda \leq \sqrt{n} / 2, n \geq 39 .
$$

Для частных случаев $n=2,3,4$ доказано более строгое неравенство

$$
\mathbb{P}\left\{D_{n}^{+} \geq \lambda\right\} \leq e^{-2 \lambda^{2}-4 \lambda^{4} / 9 n}, \lambda \geq 0 .
$$

Доказательство неравенства (2) базируется на следующей теореме.

Теорема. Пусть - случайная величина, имеющая биномиальное распределение

$$
\mathbb{P}\{\xi=k\}=\frac{n !}{k !(n-k) !} p^{k}(1-p)^{n-k}, 0 \leq p \leq 1, k=0, \ldots, n, n \in \mathbb{N} .
$$

Следующее неравенство справедливо для любых $\varepsilon, 0 \leq \varepsilon \leq q=1-p$ :

$$
\mathbb{P}\{\xi-n p>n \varepsilon\} \leq \exp \left\{-\frac{\varepsilon^{2}}{2(p+\varepsilon / 3)(q-\varepsilon / 3)}-\frac{1}{36} \varepsilon^{4}\right\} .
$$

Доказательство (3) для $n=2,3,4$ основано на использовании теоремы БуданаФурье о количестве вещественных корней многочлена

$$
P(x)=a_{n} x^{n}+a_{n-1} x^{n-1}+\cdots+a_{1} x+a_{0}, a_{n} \neq 0, x \in \mathbb{R}=(-\infty, \infty),
$$


степени $n \in \mathbb{N}$ с вещественными коэффициентами.

Пусть даны вещественные числа $c_{1}, \ldots, c_{n}$, среди которых некоторые, скажем, $c_{k_{1}}, \ldots, c_{k_{m}}$, не равны нулю. Обозначим через $W\left(c_{1}, \ldots, c_{n}\right)$ количество изменений знака в последовательности $c_{k_{1}}, \ldots, c_{k_{m}}$, если $m>1$, и $W\left(c_{1}, \ldots, c_{n}\right)=0$, если $m=1$. Число $W\left(c_{1}, \ldots, c_{n}\right)$ называется количеством изменений знака в последовательности чисел $c_{1}, \ldots, c_{n}$.

Теорема Будана-Фурье. Пусть даны многочлен (5) и его производные $P^{(k)}$, $k=1, \ldots, n$. Обозначим через $W(x)$ количество изменений знака в последовательности $P(x), P^{(1)}(x), \ldots, P^{(n)}(x), x \in \mathbb{R}$. Тогда для любых $a, b \in \mathbb{R}, a<b$ количество корней $P$ на полуинтервале $(a, b]$ с учетом их кратностей равно $W(a)-W(b)-2 m$, где $m$ - некое неотрицательное челое число.

\section{1. Основные результаты}

Начнем с доказательства неравенства (4). Непосредственно видно, что неравенство справедливо для $\varepsilon=0$. Если неравенство справедливо для $0<\varepsilon<q$, то оно справедливо для $\varepsilon=q$. Далее предполагается, что $0<\varepsilon<q$. Используя неравенство Маркова, получим

$\mathbb{P}\{\xi-n p>n \varepsilon\}=\mathbb{P}\left\{e^{x(\xi-n p)}>e^{x n \varepsilon}\right\} \leq e^{-x n \varepsilon} \mathbb{E} e^{x(\xi-n p)}=e^{-x n \varepsilon}\left(p e^{x q}+q e^{-x p}\right)^{n}, x \geq 0$.

Положим $x=n \ln ((p+\varepsilon) q /(q-\varepsilon) p)$. Нетрудно показать, что

$$
\mathbb{P}\{\xi-n p>n \varepsilon\} \leq e^{-h(p, \varepsilon)}, h(p, \epsilon)=(p+\varepsilon) \ln \left(\frac{p+\varepsilon}{p}\right)+(q-\varepsilon) \ln \left(\frac{q-\varepsilon}{q}\right) .
$$

Рассмотрим функции $h(p, \epsilon), 0<\varepsilon<q<1$, и

$$
\phi(t)=t-\frac{t^{2}}{2(1+2 t / 3)}-\ln (1+t), t \geq 0 .
$$

Так как $\phi(0)=0$ и

$$
\phi^{\prime}(t)=\frac{t^{3}}{9(1+2 t / 3)^{2}(1+t)}>0
$$

для любого положительного $t$, то функция $\phi(t), t \geq 0$, положительна, непрерывна, не убывает. Легко видеть, что

$$
\lim _{t \rightarrow \infty} \frac{\phi(t)}{t}=\frac{1}{4}
$$

Далее докажем следующее неравенство

$$
h(p, \varepsilon) \geq \frac{\varepsilon^{2}}{2(p+\varepsilon / 3)(q-\varepsilon / 3)}+\frac{1}{36} \varepsilon^{4}+\frac{\varepsilon \phi(t)}{t}, \text { где } t=\frac{\varepsilon}{q-\varepsilon} .
$$

Для этого положим $x=p+\varepsilon$ и рассмотрим разность

$$
\begin{aligned}
h(p, \varepsilon) & -\frac{\varepsilon^{2}}{2(p+\varepsilon / 3)(q-\varepsilon / 3)}-\frac{1}{36} \varepsilon^{4}-\frac{\varepsilon \phi(t)}{t}= \\
& =x \ln x-x \ln (x-\varepsilon)-\frac{1}{36} \varepsilon^{4}-\frac{\varepsilon^{2}}{2(x-2 \varepsilon / 3)}-\varepsilon, 0<\varepsilon<x<1 .
\end{aligned}
$$


Производная функции справа по переменной $\varepsilon$ равна

$$
\frac{\varepsilon^{3}}{9(x-\varepsilon)(x-2 \varepsilon / 3)^{2}}-\frac{1}{9} \varepsilon^{3}>\frac{\varepsilon^{3}}{9 x^{3}}-\frac{1}{9} \varepsilon^{3}>0 .
$$

Следовательно функция слева (8) возрастает. Так как $\phi(t) \geq 0$ и $h(p, 0)=0$, то

$$
h(p, \varepsilon) \geq \frac{\varepsilon^{2}}{2(p+\varepsilon / 3)(q-\varepsilon / 3)}+\frac{1}{36} \varepsilon^{4}+\frac{\varepsilon \phi(t)}{t} .
$$

Неравенство (4) следует (6) из (9). В действительности было доказано более строгое неравенство

$$
\mathbb{P}\{\xi-n p>n \varepsilon\} \leq \exp \left(-\frac{\varepsilon^{2}}{2(p+\varepsilon / 3)(q-\varepsilon / 3)}-\frac{\varepsilon^{4}}{36}-\frac{\varepsilon \phi(t)}{t}\right), t=\varepsilon /(q-\varepsilon) .
$$

Для доказательства неравенства (2) нам понадобится следующее равенство

$$
e^{-2 \lambda^{2}}=\int_{0}^{1} f_{\lambda}(s) d s, f_{\lambda}(s)=\frac{\lambda}{\sqrt{2 \pi}} \frac{1}{\sqrt{s^{3}(1-s)}} e^{-\lambda^{2} / 2 s(1-s)} .
$$

Это равенство может быть получено в качестве следствия глубокого результата в теории стохастических процессов. Здесь предлагается его непосредственное доказательство. Для начала покажем, что функция

$$
g(\lambda)=\frac{|\lambda|}{\pi} \int_{0}^{1} \frac{1}{\sqrt{s^{3}(1-s)}} e^{-\lambda^{2} /(2 s(1-s))} d s, \lambda \in \mathbb{R},
$$

является плотностью нормальной случайной величины $\xi$ с $\mathbb{E} \xi=0$ и $\mathbb{E} \xi^{2}=1 / 4$. По теореме Фуббини для любого целого $k \geq 0$ выполняется равенство

$$
\int_{-\infty}^{\infty}|\lambda|^{2 k} g(\lambda) d \lambda=\int_{0}^{1} \frac{2}{s \sqrt{2 \pi}} \int_{-\infty}^{\infty}|\lambda|^{2 k+1} \frac{1}{\sqrt{2 \pi s(1-s)}} e^{-\lambda^{2} /(2 s(1-s))} d \lambda d s .
$$

Внутренний интеграл равен $\pi^{-1 / 2} 2^{k+1 / 2}(s(1-s))^{k+1 / 2} k$ !. Это следует из того, что он равен математическому ожиданию $\mathbb{E}|\eta|^{2 k+1}$ нормально распределенной случайной величины $\eta$ с $\mathbb{E} \eta=0$ и $\mathbb{E} \eta^{2}=s(1-s)$. Предыдущее равенство принимает следующий вид

$$
\int_{-\infty}^{\infty}|\lambda|^{2 k} g(\lambda) d \lambda=\frac{2^{k+1} k !}{\pi} \int_{0}^{1} s^{k-1 / 2}(1-s)^{k+1 / 2} d s
$$

Интеграл в правой части есть $B$-функция $B(k+1 / 2, k+3 / 2)$. Он может быть записан с помощью Г-функций:

$$
\int_{0}^{1} s^{k-1 / 2}(1-s)^{k+1 / 2} d s=B(k+1 / 2, k+3 / 2)=\frac{\Gamma(k+1 / 2) \Gamma(k+3 / 2)}{\Gamma(2 k+2)} .
$$

Так как $\Gamma(1 / 2) \Gamma(1 / 2)=\pi$ и $\Gamma(\alpha+1)=\alpha \Gamma(\alpha)$ для любого $\alpha>0$, то

$$
\int_{-\infty}^{\infty}|\lambda|^{2 k} g(\lambda) d \lambda=\frac{2^{k+1}}{\pi} \frac{\Gamma(k+1 / 2) \Gamma(k+3 / 2)}{\Gamma(2 k+2)}= \begin{cases}1, & \text { при } k=0, \\ \frac{1}{4^{k}}(2 k-1) ! !, & \text { при } k \geq 1,\end{cases}
$$


где $(2 k-1) ! !=1 \cdot 3 \cdots(2 k-1)$ является произведением нечетных натуральных чисел от 1 до $2 k-1$. Из равенства для $k=0$ следует, что функция $g(\lambda), \lambda \in \mathbb{R}$, является симметричной функцией плотности. Равенства для $k \geq 1$ показывают, что все четные моменты случайной величины $\xi$ с плотностью $g$ совпадают с четными моментами нормальной случайной величины $\xi^{\prime}$ с $\mathbb{E} \xi^{\prime}=0$ и $\mathbb{E}\left|\xi^{\prime}\right|^{2}=1 / 4$. Любая нормальная случайная величина определяется своими моментами. Поэтому справедливо равенство $g(\lambda)=\sqrt{2 / \pi} \exp \left\{-2 \lambda^{2}\right\}$ для любых $\lambda \in \mathbb{R}$ и, следовательно,

$$
\int_{0}^{1} f_{\lambda}(s) d s=\sqrt{\frac{\pi}{2}} g(\lambda)=\sqrt{\frac{\pi}{2}} \sqrt{\frac{2}{\pi}} e^{-2 \lambda^{2}}=e^{-2 \lambda^{2}}, \lambda \geq 0 .
$$

Неравенство (10) доказано.

Доказательство (2) основано на идеях Массарта [3]. Обозначив $j=n-k$, выражение (1) для $0<\lambda<\sqrt{n}$ может быть переписано в следующем виде

$$
\begin{aligned}
& \mathbb{P}\left\{D_{n}^{+}>\lambda\right\}=\sum_{0 \leq j<n-\lambda \sqrt{n}} p_{\lambda, n}(j), \\
& p_{\lambda, n}(j)=\lambda \sqrt{n}(j+\lambda \sqrt{n})^{j-1}(n-j-\lambda \sqrt{n})^{n-j} n^{-n}\left(\begin{array}{c}
n \\
j
\end{array}\right) .
\end{aligned}
$$

Лемма 1. Пусть $v_{n}\left(s^{\prime}\right)=1 /\left(s^{\prime}\left(s^{\prime 2}-1 /(4 n)\right)\right)$. Тогда при любых положительных $\varepsilon, s, s^{\prime}=1-s$ таких, что $n \varepsilon \geq 2 u n s^{\prime} \geq 1$, справедливо следующее неравенство

$$
\left(1+\frac{2 \varepsilon}{3 s^{\prime}}\right)^{-\frac{1}{2}} \leq\left(1-\frac{\varepsilon}{3 s^{\prime}}+\frac{\varepsilon^{2}}{6 s^{\prime 2}}\right) \exp \left(-\frac{\varepsilon^{2} v_{n}\left(s^{\prime}\right)}{24 n}\right) .
$$

Доказательство. Рассмотрим функцию $\rho(x)=1+\alpha x-\exp (2 \beta x)$, где $\alpha=135 / 32, \beta=0.826$. Заметим, что $\rho(x)$ вогнута и неотрицательна на сегменте $[0,1]$, так как $\rho(0)=0$ и $\rho(1) \geq 1.3 \cdot 10^{-3}$. Следовательно

$$
\exp \left(2 \beta x^{3}\right) \leq 1+\alpha x^{3} \leq 1+\alpha x^{3}+\frac{x^{3}(5-12 x)^{2}}{32}=\left(1-x+\frac{3 x^{2}}{2}\right)^{2}(1+2 x)
$$

Используем неравенство выше с $x=\varepsilon /\left(3 s^{\prime}\right)$. Тогда для доказательства (11) остается показать, что

$$
\frac{\beta \varepsilon^{3}}{27 s^{\prime 3}} \geq \frac{\varepsilon^{2} v_{n}\left(s^{\prime}\right)}{24 n}
$$

что равносильно

$$
\frac{8 \beta}{9} \geq\left(n \varepsilon\left(1-\left(2 n s^{\prime}\right)^{-2}\right)\right)^{-1}
$$

Так как $n \varepsilon \geq 2$ и $n s^{\prime} \geq 1$, то выполняются неравенства

$$
\left(n \varepsilon\left(1-\left(2 n s^{\prime}\right)^{-2}\right)\right)^{-1} \leq \frac{2}{3}<\frac{8 \beta}{9} .
$$

Это завершает доказательство леммы. 
Лемма 2. Пусть $0 \leq j<n-\lambda \sqrt{n}, \varepsilon=\lambda / \sqrt{n}, s=2 \varepsilon / 3+j / n, s^{\prime}=1-s$. Если $n \varepsilon \geq 2, m o$

$$
p_{\lambda, n}(j) \leq \frac{1}{n}\left(1-\frac{\varepsilon}{3 s^{\prime}}+\frac{\varepsilon^{2}}{6 s^{\prime 2}}\right) \exp \left(\frac{0.4}{n s}-\frac{\varepsilon^{2}}{24 n}\left(v_{n}(s)+v_{n}\left(s^{\prime}\right)\right) f_{\lambda}(s) e^{-\lambda^{4} / 36 n},\right.
$$

где $v_{n}(s)=1 /\left(s\left(s^{2}-1 /(4 n)\right)\right)$.

Доказательство. Рассмотрим случай $j=0$. В этом случае $s=2 \varepsilon / 3$. Напомним, что $\varepsilon=\lambda / \sqrt{n}$ и $q=1-p$. Из (7) и (9) следует, что

$$
-\ln (1-\varepsilon)=\lim _{q \rightarrow \varepsilon} h(p, \varepsilon) \geq \frac{\varepsilon^{2}}{2(1-2 \varepsilon / 3) 2 \varepsilon / 3}+\frac{\varepsilon}{4}+\frac{\varepsilon^{4}}{36}=\frac{\varepsilon}{4}+\frac{\varepsilon^{2}}{2 s s^{\prime}}+\frac{\varepsilon^{4}}{36} .
$$

Обозначим

$$
H(v)=\frac{3 \ln (3 / 2)}{2}-\frac{\ln (2 \pi)}{2}+\frac{v}{4}+\frac{0.4}{v}-\frac{\ln v}{2}, v>1
$$

Величину $p_{\lambda, n}(0)$ можно оценить следующим образом

$$
\begin{aligned}
p_{\lambda, n}(0) & =(1-\varepsilon)^{n}=e^{n \ln (1-\varepsilon)} \leq \exp \left(-\frac{n \varepsilon}{4}-\frac{\lambda^{2}}{2 s s^{\prime}}-\frac{\lambda^{4}}{36 n}\right) \leq \\
& \leq \frac{\lambda}{n \sqrt{2 \pi}} s^{-3 / 2} \exp \left(\frac{0.4}{n \varepsilon}\right) \exp \left(-\frac{\lambda^{2}}{2 s s^{\prime}}\right) \exp (-H(n \varepsilon)) e^{-\lambda^{4} / 36 n} .
\end{aligned}
$$

Функция $H(v), v>1$ положительна. Она принимает минимальное значение в точке $v_{0}=1+\sqrt{2.6}$ и $H\left(v_{0}\right) \geq 1.5 \cdot 10^{-2}$. Поэтому

$$
\begin{aligned}
p_{\lambda, n}(0) & \leq \frac{\lambda}{n \sqrt{2 \pi}} s^{-3 / 2} \exp \left(\frac{0.4}{n \varepsilon}\right) \exp \left(-\frac{\lambda^{2}}{2 s s^{\prime}}\right) e^{-\lambda^{4} / 36 n} \leq \\
& \leq \frac{1}{n}\left(1+\frac{2 \varepsilon}{3 s^{\prime}}\right)^{-1 / 2} \exp \left(\frac{0.4}{n \varepsilon}\right) f_{\lambda}(s) e^{-\lambda^{4} / 36 n} .
\end{aligned}
$$

Так как $\varepsilon n \geq 2$, то справедливы неравенства

$$
\begin{aligned}
& \frac{\varepsilon^{2} v_{n}(s)}{24 n} \leq \frac{1}{16 n \varepsilon(4 / 9-1 / 16)} \leq \frac{9}{55 n \varepsilon}, \\
& \frac{0.4}{n \varepsilon}+\frac{\varepsilon^{2} v_{n}(s)}{24 n} \leq \frac{0.4+9 / 55}{n \varepsilon} \leq \frac{0.4}{n s} .
\end{aligned}
$$

Из этих неравенств и предыдущей оценки для $p_{\lambda, n}(0)$ следует, что

$$
p_{\lambda, n}(0) \leq \frac{1}{n}\left(1+\frac{2 \varepsilon}{3 s^{\prime}}\right)^{-1 / 2} \exp \left(\frac{0.4}{n s}-\frac{\varepsilon^{2} v_{n}(s)}{24 n}\right) f_{\lambda}(s) e^{-\lambda^{4} / 36 n} .
$$

Это неравенство вместе с (11) влекут неравенство (12) для $j=0$.

Предположим теперь, что $1 \leq j<n-\lambda \sqrt{n}$. По формуле Стирлинга (см. [4], стр. 54) выполняются неравенства

$$
\sqrt{2 \pi} n^{n+1 / 2} e^{-n} e^{-1 /(12 n)}<n !<\sqrt{2 \pi} n^{n+1 / 2} e^{-n} e^{-1 /(12 n+1)} .
$$


В силу правого неравенства справедлива следующая оценка

$$
\left(\begin{array}{c}
n \\
j
\end{array}\right) \leq \frac{1}{\sqrt{2 \pi}} \sqrt{\frac{n}{j(n-j)}} \frac{n^{n}}{j^{j}(n-j)^{n-j}} C_{j}, C_{j}=e^{-1 /(12 j+1)}, j \geq 1 .
$$

Это, в свою очередь, ведет к оценке

$$
p_{\lambda, n}(j) \leq \frac{\lambda}{\sqrt{2 \pi}} \frac{n}{\sqrt{j(n-j)}(j+\lambda \sqrt{n})}\left(\frac{j+\lambda \sqrt{n}}{j}\right)^{j}\left(\frac{n-j-\lambda \sqrt{n}}{n-j}\right)^{n-j} C_{j} .
$$

Положим $\varepsilon=\lambda / \sqrt{n}, s_{j}=s=2 \varepsilon / 3+j / n$ и $s_{j}^{\prime}=s^{\prime}=1-s$. Предыдущее неравенство с помощью функции $h(\cdot, \cdot)$ из (6) преобразуется к следующему виду

$$
p_{\lambda, n}(j) \leq \frac{\lambda}{n \sqrt{2 \pi}}\left(s-\frac{2 \varepsilon}{3}\right)^{-1 / 2}\left(s+\frac{\varepsilon}{3}\right)^{-1}\left(s^{\prime}+\frac{2 \varepsilon}{3}\right)^{-1 / 2} e^{-n h\left(s^{\prime}-\varepsilon / 3, \varepsilon\right)} C_{j} .
$$

Определим функцию

$$
\psi(t)=-\ln (1+t)+\frac{3}{2} \ln \left(1+\frac{2 t}{3}\right), t \geq 0
$$

Из неравенства (9) с $p=s^{\prime}-\varepsilon / 2$ следует, что

$$
\begin{aligned}
p_{\lambda, n}(j) & \leq \frac{1}{n}\left(1+\frac{2 \varepsilon}{3 s^{\prime}}\right)^{-1 / 2} \frac{s^{3 / 2}}{\sqrt{s-2 \varepsilon / 3}(s+\varepsilon / 3)} C_{j} e^{-n \varepsilon \phi(t) / t} f_{\lambda}(s) e^{-\lambda^{4} / 36 n}= \\
& =\frac{1}{n}\left(1+\frac{2 \varepsilon}{3 s^{\prime}}\right)^{-1 / 2} \frac{s^{3 / 2}}{\sqrt{s-2 \varepsilon / 3}(s+\varepsilon / 3)} C_{j} e^{-n \varepsilon \phi(t) / t+\psi(t)} f_{\lambda}(s) e^{-\lambda^{4} / 36 n} .
\end{aligned}
$$

Это неравенство является более строгой версией неравенства (2.7) из [3]. Неравенство (2.7) из статьи Массарта получается путем замены $e^{-\lambda^{4} / 36 n}$ на единицу. Теперь для доказательства (12) при $1 \leq j<n-\lambda \sqrt{n}$ можно пошагово повторить действия Массарта [3].

Докажем, что (2) следует из (12) для $n \geq 39, \gamma n^{-1 / 6} \leq \lambda \leq \sqrt{n} / 2$. По аналогии с [3] получим

$$
e^{2 \lambda^{2}+\lambda^{4} / 36 n} \mathbb{P}\left\{D_{m}^{+}>\lambda\right\} \leq J_{0,1}(\lambda)-\frac{\varepsilon}{3} J_{1,1}(\lambda)+\frac{\varepsilon^{2}}{6} J_{2,1}(\lambda)+\frac{\mu}{n} J_{2,0}(\lambda)-\frac{2 \mu}{3 n} J_{2,1}+\frac{\varepsilon^{2} \mu}{6 n} J_{2,2}(\lambda),
$$

где $\mu=0.4345$,

$$
J_{a, b}(\lambda)=\frac{\lambda}{\sqrt{2 \pi}} e^{2 \lambda^{2}} \int_{0}^{1} u^{-1 / 2-a}(1-u)^{-1 / 2-b} e^{-\lambda^{2} /(2 u(1-u))} d u .
$$

Из (10) получим $1=J_{1,0}(\lambda)=1 / 2 \cdot\left(J_{1,0}(\lambda)+J_{0,1}(\lambda)\right)=1 / 2 \cdot J_{1,1}(\lambda)$. С помощью (10) нетрудно доказать, что $J_{2,2}(\lambda) / 2=J_{2,1}(\lambda)=4+\lambda^{-2}, J_{2,0}(\lambda)=2+\lambda^{-2}$. Из предыдущего неравенства следует, что

$$
\begin{aligned}
& \frac{3 \sqrt{n}}{2 \lambda}\left(e^{2 \lambda^{2}+\lambda^{4} / 36 n} \mathbb{P}\left(D_{n}^{+}>\lambda\right)-1\right) \leq \\
& \leq-1+\left(\lambda+\frac{1}{4 \lambda}+\frac{3 \mu}{\lambda}+\frac{3 \mu}{2 \lambda^{3}}\right) n^{-1 / 2}-\frac{\mu}{2}\left(4+\frac{1}{\lambda^{2}}\right) n^{-1}+\frac{\mu}{2}\left(4 \lambda+\frac{1}{\lambda}\right) n^{-3 / 2} .
\end{aligned}
$$


Массарт [3] доказал, что правая часть выражения выше отрицательна. Это завершает доказательство (2).

\section{2. Случай малых объемов выборок}

Доказать (3) нам поможет следующая лемма.

Лемма 3. Справедливо следующее неравенство

$$
-\ln (1-\varepsilon) \geq 2 \varepsilon^{2}+\frac{4}{9} \varepsilon^{4}, 0 \leq \varepsilon<1 .
$$

Доказательство. Достаточно доказать, что функция $f(\varepsilon)=\ln (1-\varepsilon)+2 \varepsilon^{2}+4 \varepsilon^{4} / 9$ принимает неположительные значения для всех $\varepsilon \in[0,1)$. Производная данной функции равна

$$
f^{(1)}(\varepsilon)=\frac{P(\varepsilon)}{1-\varepsilon}, P(\varepsilon)=-\frac{16}{9} \varepsilon^{4}+\frac{16}{9} \varepsilon^{3}-4 \varepsilon^{2}+4 \varepsilon-1 .
$$

Первая и вторая производная функции $P(\varepsilon), 0 \leq \varepsilon \leq 1$, имеют следующий вид

$$
P^{(1)}(\varepsilon)=-\frac{64}{9} \varepsilon^{3}+\frac{48}{9} \varepsilon^{2}-8 \varepsilon+4, P^{(2)}(\varepsilon)=-\frac{64}{3} \varepsilon^{2}+\frac{32}{3} \varepsilon-8 .
$$

Вторая производная $P^{(2)}(\varepsilon)=-(8 \varepsilon-3)^{2} / 3-5$ отрицательна. Следовательно $P^{(1)}$ убывает, и функция $P$ вогнута. Т.к. $P(0)=P(1)=-1$ и $P(0.6)=0.1136$, существуют $0<\varepsilon_{1}<\varepsilon_{2}<1$, такие, что функция $P(\varepsilon)$ отрицательна при $\varepsilon \in\left[0, \varepsilon_{1}\right) \cup\left(\varepsilon_{2}, 1\right]$ и положительна для $\varepsilon \in\left(\varepsilon_{1}, \varepsilon_{2}\right)$. Получим, что $f(\varepsilon)$ убывает на $\left[0, \varepsilon_{1}\right) \cup\left(\varepsilon_{2}, 1\right]$ и возрастает на $\left(\varepsilon_{1}, \varepsilon_{2}\right)$. Поэтому функция $f$ принимает максимальное значение при $\varepsilon=0$ или $\varepsilon=\varepsilon_{2}$. Заметим, что $f(0)=0$. Покажем, что $f\left(\varepsilon_{2}\right) \leq 0$. Так как $P(14 / 20)=43 / 1875>0$ и $P(15 / 20)=-0.0625<0$, получим, что $14 / 20<\varepsilon_{2}<15 / 20$. По формуле Тэйлора существует $\varepsilon^{\prime} \in\left[14 / 20, \varepsilon_{2}\right]$, что

$$
f\left(\varepsilon_{2}\right)=f(14 / 20)+\frac{P\left(\varepsilon^{\prime}\right)}{1-\varepsilon^{\prime}}\left(\varepsilon_{2}-14 / 20\right) \leq f(14 / 20)+\frac{P(14 / 20)}{1-15 / 20}(15 / 20-14 / 20)<0 .
$$

Это доказывает, что $f$ отрицательна. Неравенство (13) доказано.

Утверждение 1. Неравенство (3) справедливо при $n=2$.

Доказательство. Из (1) и (13) для $(n-1) / \sqrt{n} \leq \lambda<\sqrt{n}, n \geq 1$, следует, что

$$
\mathbb{P}\left\{D_{n}^{+}>\lambda\right\}=\left(1-\frac{\lambda}{\sqrt{n}}\right)^{n} \leq e^{-2 \lambda^{2}-4 \lambda^{4} / 9} .
$$

Осталось рассмотреть промежуток $0<\lambda<1 / \sqrt{2}$. Обозначим $f_{2}(\lambda)=\mathbb{P}\left\{D_{2}^{+}>\lambda\right\}$. Достаточно доказать, что

$$
\left(\ln \left(f_{2}(\lambda) e^{2 \lambda^{2}+2 \lambda^{4} / 9}\right)\right)^{\prime}=\frac{f_{2}^{\prime}(\lambda)+\left(4 \lambda+8 \lambda^{3} / 9\right) f_{2}(\lambda)}{f_{2}(\lambda)} \leq 0 .
$$


При $0<\lambda<1 / \sqrt{2}$ получим

$$
\begin{array}{r}
f_{2}(\lambda)=-\frac{1}{2} \lambda^{2}-\frac{1}{\sqrt{2}} \lambda+1 ; f_{2}^{\prime}(\lambda)=-\lambda-\frac{1}{\sqrt{2}} ; \\
\begin{array}{r}
f_{2}^{\prime}(\lambda)+\left(4 \lambda+\frac{8}{9} \lambda^{3}\right) f_{2}(\lambda)=-\frac{4}{9} \lambda^{5}-\frac{4 \sqrt{2}}{9} \lambda^{4}-\frac{10}{9} \lambda^{3}-2 \sqrt{2} \lambda^{2}+3 \lambda-\frac{1}{\sqrt{2}} \leq \\
\leq-\frac{10}{9} \lambda^{3}-2 \sqrt{2} \lambda^{2}+3 \lambda-\frac{1}{\sqrt{2}} .
\end{array}
\end{array}
$$

Рассмотрим многочлен $P_{2}(\lambda)=-10 \lambda^{3} / 9-2 \sqrt{2} \lambda^{2}+3 \lambda-1 / \sqrt{2}, \lambda \in \mathbb{R}$, и его производную

$$
P_{2}^{(1)}(\lambda)=-\frac{10}{3} \lambda^{2}-4 \sqrt{2} \lambda+3=-\frac{10}{3}\left(\lambda+\frac{3}{\sqrt{2}}\right)\left(\lambda-\frac{3}{5 \sqrt{2}}\right) .
$$

Функция $P_{2}(\lambda), 0 \leq \lambda \leq 1 / \sqrt{2}$, принимает максимальное значение в точке $\lambda=3 / 5 \sqrt{2}$. Так как $P_{2}(3 / 5 \sqrt{2})=-1 / 25 \sqrt{2}<0$, то $P_{2}(\lambda) \leq 0$ для всех $0 \leq \lambda \leq 1 / \sqrt{2}$. Следовательно, неравенство (14) справедливо. Утверждение доказано.

Утверждение 2. Неравенство (3) справедливо при $n=3$.

Доказательство. В силу (14) достаточно доказать неравенство только для $0<\lambda<2 / \sqrt{3}$. Обозначим $f_{3}(\lambda)=\mathbb{P}\left\{D_{3}^{+}>\lambda\right\}$ и рассмотрим следующую функцию

$$
\left(\ln \left(f_{3}(\lambda) e^{2 \lambda^{2}+4 \lambda^{4} / 27}\right)\right)^{\prime}=\frac{f_{3}^{\prime}(\lambda)+\left(4 \lambda+16 \lambda^{3} / 27\right) f_{3}(\lambda)}{f_{3}(\lambda)} .
$$

При $0<\lambda<1 / \sqrt{3}$ имеем

$$
\begin{gathered}
f_{3}(\lambda)=-\frac{1}{3 \sqrt{3}} \lambda^{3}-\frac{2}{3} \lambda^{2}-\frac{1}{\sqrt{3}} \lambda+1 ; f_{3}^{\prime}(\lambda)=-\frac{1}{\sqrt{3}} \lambda^{2}-\frac{4}{3} \lambda-\frac{1}{\sqrt{3}} ; \\
f_{3}^{\prime}(\lambda)+\left(4 \lambda+\frac{16 \lambda^{3}}{27}\right) f_{3}(\lambda)=-\frac{16}{81 \sqrt{3}} \lambda^{6}-\frac{32}{81} \lambda^{5}-\frac{52}{27 \sqrt{3}} \lambda^{4}-\frac{56}{27} \lambda^{3}-\frac{5}{\sqrt{3}} \lambda^{2}+\frac{8}{3} \lambda-\frac{1}{\sqrt{3}} \leq \\
\leq-\frac{5}{\sqrt{3}} \lambda^{2}+\frac{8}{3} \lambda-\frac{1}{\sqrt{3}}=-\frac{5}{\sqrt{3}}\left(\lambda-\frac{1}{\sqrt{3}}\right)\left(\lambda-\frac{\sqrt{3}}{5}\right)<0 .
\end{gathered}
$$

Функция (16) отрицательна, следовательно

$$
f_{3}(\lambda) e^{2 \lambda^{2}+4 \lambda^{4} / 27} \leq f_{3}(0)=1, \lambda \in\left[0, \frac{1}{\sqrt{3}}\right) .
$$

Для $\lambda \in[1 / \sqrt{3}, 2 / \sqrt{3})$ имеем

$$
\begin{aligned}
& f_{3}(\lambda)=\frac{2}{3 \sqrt{3}} \lambda^{3}-\frac{1}{3} \lambda^{2}-\frac{5}{3 \sqrt{3}} \lambda+1 ; f_{3}^{\prime}(\lambda)=\frac{2}{\sqrt{3}} \lambda^{2}-\frac{2}{3} \lambda-\frac{5}{3 \sqrt{3}} \\
& f_{3}^{\prime}(\lambda)+\left(4 \lambda+\frac{16 \lambda^{3}}{9 n}\right) f_{3}(\lambda)=\frac{32}{81 \sqrt{3}} \lambda^{6}-\frac{16}{81} \lambda^{5}+\frac{136}{81 \sqrt{3}} \lambda^{4}-\frac{20}{27} \lambda^{3}-\frac{14}{3 \sqrt{3}} \lambda^{2}+\frac{10}{3} \lambda-\frac{5}{3 \sqrt{3}} .
\end{aligned}
$$


Рассмотрим многочлен $P_{3}(\lambda)=f_{3}^{\prime}(\lambda)+\left(4 \lambda+16 \lambda^{3} /(27)\right) f_{3}(\lambda), \lambda \in \mathbb{R}$. Для применения теоремы Будана-Фурье найдем его производные

$$
\begin{aligned}
& P_{3}^{(1)}(\lambda)=\frac{64}{27 \sqrt{3}} \lambda^{5}-\frac{80}{81} \lambda^{4}+\frac{544}{81 \sqrt{3}} \lambda^{3}-\frac{20}{9} \lambda^{2}-\frac{28}{3 \sqrt{3}} \lambda+\frac{10}{3} \\
& P_{3}^{(2)}(\lambda)=\frac{320}{27 \sqrt{3}} \lambda^{4}-\frac{320}{81} \lambda^{3}+\frac{544}{27 \sqrt{3}} \lambda^{2}-\frac{40}{9} \lambda-\frac{28}{3 \sqrt{3}} ; \\
& P_{3}^{(3)}(\lambda)=\frac{1280}{27 \sqrt{3}} \lambda^{3}-\frac{320}{27} \lambda^{2}+\frac{1088}{27 \sqrt{3}} \lambda-\frac{40}{9} \\
& P_{3}^{(4)}(\lambda)=\frac{1280}{9 \sqrt{3}} \lambda^{2}-\frac{640}{27} \lambda+\frac{1088}{27 \sqrt{3}} \\
& P_{3}^{(5)}(\lambda)=\frac{2560}{9 \sqrt{3}} \lambda-\frac{640}{27} .
\end{aligned}
$$

Непосредственные вычисления показывают, что

$$
\begin{array}{lll}
+, P_{3}(1 / \sqrt{3})=\frac{95}{2187 \sqrt{3}}>0 ; & +, P_{3}(2 / \sqrt{3})=\frac{47}{2187 \sqrt{3}}>0 \\
+, P_{3}^{(1)}(1 / \sqrt{3})=\frac{50}{243}>0 ; & +, P_{3}^{(1)}(2 \sqrt{3})=\frac{854}{729}>0 ; \\
-, P_{3}^{(2)}(1 / \sqrt{3})=-\frac{572}{81 \sqrt{3}}<0 ; & +, P_{3}^{(2)}(2 / \sqrt{3})=\frac{4660}{243 \sqrt{3}}>0 \\
+, P_{3}^{(3)}(1 \sqrt{3})=\frac{2504}{243}>0 ; & +, P_{3}^{(3)}(2 / \sqrt{3})=\frac{11848}{243}>0 \\
+, P_{3}^{(4)}(1 \sqrt{3})=\frac{64}{\sqrt{3}}>0 ; & +, P_{3}^{(4)}(2 \sqrt{3})=\frac{4928}{27 \sqrt{3}}>0 \\
+, P_{3}^{(5)}(1 / \sqrt{3})=\frac{640}{9}>0 ; & +P_{3}^{(5)}(2 / \sqrt{3})=\frac{4480}{27}>0 \\
+, P_{3}^{(6)}(1 \sqrt{3})=\frac{2560}{9 \sqrt{3}}>0 ; & +, P_{3}^{(6)}(2 \sqrt{3})=\frac{2560}{9 \sqrt{3}}>0
\end{array}
$$

По теореме Будана-Фурье многочлен $P_{3}^{(3)}(\lambda)$ положителен на сегменте $\lambda \in[1 / \sqrt{3}, 2 / \sqrt{3}]$. Следовательно функция $P_{3}^{(1)}(\lambda)$ выпукла на сегменте $[1 / \sqrt{3}, 2 \sqrt{3}]$. Так как

$$
\begin{gathered}
P_{3}^{(1)}(1 / \sqrt{3})=95 / 2187 \sqrt{3}>0, P_{3}^{(1)}(\sqrt{3} / 2)=-10 / 27<0, \\
P_{3}^{(1)}(2 / \sqrt{3})=47 / 2187 \sqrt{3}>0,
\end{gathered}
$$

то существуют $1 / \sqrt{3}<\lambda_{1}<\lambda_{2}<2 / \sqrt{3}, \lambda_{1}<\sqrt{3} / 2$, такие что $P_{3}^{(1)}\left(\lambda_{1}\right)=$ $=P_{3}^{(1)}\left(\lambda_{2}\right)=0$. Это значит, что функция $P_{3}(\lambda)$ возрастает при $\left[1 / \sqrt{3}, \lambda_{1}\right] \cup\left[\lambda_{2}, 2 / \sqrt{3}\right]$ и убывает при $\left[\lambda_{1}, \lambda_{2}\right]$. Так как $P_{3}^{(1)}(2 / \sqrt{3})=854 / 729>0$, то из (16) следует, что максимальное значение функции $f_{3}(\lambda) e^{2 \lambda^{2}+4 \lambda^{4} / 27}, \lambda \in\left[\lambda_{2}, 2 / \sqrt{3}\right]$, достигается в точке $\lambda=2 / \sqrt{3}$ и равно $f_{3}(2 / \sqrt{3}) e^{2(2 / \sqrt{3})^{2}+4(2 / \sqrt{3})^{4} / 27}=0.693644<1$. Функция $f_{3}(\lambda) e^{2 \lambda^{2}+4 \lambda^{4} / 27}, \lambda \in\left[1 / \sqrt{3}, \lambda_{2}\right]$ принимает свое максимальное значение в точке $\lambda_{1}$. 
Заметим, что $1 / \sqrt{3}<39 / 50<40 / 50<\sqrt{3} / 2$ и

$$
\begin{aligned}
& P(39 / 50)=\frac{21400873}{9765625}-\frac{5559684907}{1464843750 \sqrt{3}}>0.00016>0 ; \\
& P(40 / 50)=\frac{562616}{253125}-\frac{1629301}{421875 \sqrt{3}}<-0.007<0 .
\end{aligned}
$$

Следовательно $39 / 50<\lambda_{1}<40 / 50$. Поэтому $f_{3}(\lambda) e^{2 \lambda^{2}+4 \lambda^{4} / 27}$ возрастает на $\left[39 / 50, \lambda_{1}\right]$ и убывает на $\left[\lambda_{1}, 40 / 50\right]$. Так как функция $f(\lambda)$ убывает, получим

$$
f\left(\lambda_{1}\right) e^{2 \lambda_{1}^{2}+4 \lambda_{1}^{4} / 27} \leq f(39 / 50) e^{2(40 / 50)^{2}+4(40 / 50)^{2} / 27}=0.488788<1 .
$$

Это завершает доказательство утверждения.

Утверждение 3. Неравенство (3) справедливо при $n=4$.

Доказательство. В силу (14) достаточно доказать неравенство только для $0 \leq \lambda<3 / 2$. Рассмотрим функцию $f_{4}(\lambda)=\mathbb{P}\left\{D_{4}^{+}>\lambda\right\}$ и производную

$$
\left(\ln \left(f_{4}(\lambda) e^{2 \lambda^{2}+\lambda^{4} / 9}\right)^{\prime}=\frac{f_{4}^{\prime}(\lambda)+\left(4 \lambda+4 \lambda^{3} / 9\right) f_{4}(\lambda)}{f_{4}(\lambda)} .\right.
$$

При $0<\lambda<1 / 2$ имеем

$$
\begin{aligned}
& f_{4}(\lambda)=-\frac{1}{16} \lambda^{4}-\frac{3}{8} \lambda^{3}-\frac{3}{4} \lambda^{2}-\frac{1}{2} \lambda+1 ; f_{4}^{\prime}(\lambda)=-\frac{1}{4} \lambda^{3}-\frac{9}{8} \lambda^{2}-\frac{3}{2} \lambda-\frac{1}{2} ; \\
& f_{4}^{\prime}(\lambda)+\left(4 \lambda+\frac{4}{9} \lambda^{3}\right) f_{4}(\lambda)=-\frac{1}{36} \lambda^{7}-\frac{1}{6} \lambda^{6}-\frac{7}{12} \lambda^{5}-\frac{31}{18} \lambda^{4}-\frac{101}{36} \lambda^{3}-\frac{25}{8} \lambda^{2}+\frac{5}{2} \lambda-\frac{1}{2} \leq \\
& \leq-\frac{25}{8} \lambda^{2}+\frac{5}{2} \lambda-\frac{1}{2}=-\frac{1}{8}(5 \lambda-2)^{2} \leq 0 .
\end{aligned}
$$

Из (17) следует, что

$$
f_{4}(\lambda) e^{2 \lambda^{2}+\lambda^{4} / 9} \leq f_{4}(0)=1, \text { при } 0 \leq \lambda<1 / 2 .
$$

Если $1 / 2 \leq \lambda<1$, то

$$
\begin{aligned}
& f_{4}(\lambda)=\frac{3}{16} \lambda^{4}+\frac{1}{4} \lambda^{3}-\frac{9}{16} \lambda^{2}-\frac{25}{32} \lambda+1 ; f_{4}^{\prime}(\lambda)=\frac{3}{4} \lambda^{3}+\frac{3}{4} \lambda^{2}-\frac{9}{8} \lambda-\frac{25}{32} \\
& f_{4}^{\prime}(\lambda)+\left(4 \lambda+\frac{4}{9} \lambda^{3}\right) f_{4}(\lambda)=\frac{\lambda^{7}}{12}+\frac{\lambda^{6}}{9}+\frac{\lambda^{5}}{2}+\frac{47 \lambda^{4}}{72}-\frac{19 \lambda^{3}}{18}-\frac{19 \lambda^{2}}{8}+\frac{23 \lambda}{8}-\frac{25}{32} .
\end{aligned}
$$

Если $1 / 2 \leq \lambda \leq 14 / 25$, то

$$
f_{4}(\lambda) e^{2 \lambda^{2}+\lambda^{4} / 9} \leq f_{4}(1 / 2) e^{2(14 / 25)^{2}+(14 / 25)^{4} / 9}=0.968649<1 \text { для } 1 / 2 \leq \lambda<14 / 25 .
$$

Если $0.99 \leq \lambda<1$, то

$$
f_{4}(\lambda) e^{2 \lambda^{2}+\lambda^{4} / 9} \leq f_{4}(99 / 100) e^{2(1)^{2}+(1)^{4} / 9}<0.81<1, \frac{99}{100} \leq \lambda<1 .
$$


Рассмотрим $P_{4}(\lambda)=f_{4}^{\prime}(\lambda)+\left(2 \lambda^{2}+\lambda^{4} / 9\right) f_{4}(\lambda)$ на сегменте [14/25, 99/100]. Его производные равны

$$
\begin{aligned}
& P_{4}^{(1)}(\lambda)=\frac{7 \lambda^{6}}{12}+\frac{2 \lambda^{5}}{3}+\frac{5 \lambda^{4}}{2}+\frac{47 \lambda^{3}}{18}-\frac{19 \lambda^{2}}{6}-\frac{19 \lambda}{4}+\frac{23}{8} \\
& P_{4}^{(2)}(\lambda)=\frac{7 \lambda^{5}}{2}+\frac{10 \lambda^{4}}{3}+10 \lambda^{3}+\frac{47 \lambda^{2}}{6}-\frac{19 \lambda}{3}-\frac{19}{4} \\
& P_{4}^{(3)}(\lambda)=\frac{35 \lambda^{4}}{2}+\frac{40 \lambda^{3}}{3}+30 \lambda^{2}+\frac{47 \lambda}{3}-\frac{19}{3} \\
& P_{4}^{(4)}(\lambda)=70 \lambda^{3}+40 \lambda^{2}+60 \lambda+\frac{47}{3} \\
& P_{4}^{(5)}(\lambda)=210 \lambda^{2}+80 \lambda+60 \\
& P_{4}^{(6)}(\lambda)=420 \lambda+80 .
\end{aligned}
$$

Непосредственные вычисления показывают, что

$$
\begin{aligned}
& -, P_{4}(14 / 25)=-\frac{8476927729}{1757812500000}<0 ; \quad-, P_{4}(99 / 100)=-\frac{844091394167}{40000000000000}<0 ; \\
& -, P_{4}^{(1)}(14 / 25)=-\frac{333023813}{17578125000}<0 ; \quad+, P_{4}^{(1)}(99 / 100)=\frac{4748354581669}{400000000000}>0 ; \\
& -, P_{4}^{(2)}(14 / 25)=-\frac{139195339}{39062500}<0 ; \quad+, P_{4}^{(2)}(99 / 100)=\frac{2578000037493}{200000000}>0 ; \\
& +, P_{4}^{(3)}(14 / 25)=\frac{3729043}{234375}>0 ; \quad+, P_{4}^{(3)}(99 / 100)=\frac{8192900021}{120000000}>0 ; \\
& +, P_{4}^{(4)}(14 / 25)=\frac{694723}{9375}>0 ; \quad+, P_{4}^{(4)}(99 / 100)=\frac{54650779}{300000}>0 ; \\
& +, P_{4}^{(5)}(14 / 25)=\frac{21332}{125}>0 ; \quad+, P_{4}^{(5)}(99 / 100)=\frac{345021}{1000}>0 ; \\
& +, P_{4}^{(6)}(14 / 25)=\frac{1576}{5}>0 ; \quad \quad+, P_{4}^{(6)}(99 / 100)=\frac{2479}{5}>0 ; \\
& +, P_{4}^{(7)}(14 / 25)=420>0 ; \quad+, P_{4}^{(7)}(99 / 100)=420>0 .
\end{aligned}
$$

По теореме Будана-Фурье многочлен $P_{4}(\lambda)$ не имеет вещественных корней на отрезке $[14 / 25,99 / 100]$. Так как $P_{4}(1 / 2)<0$, то производная (17) отрицательна и, следовательно,

$$
\begin{aligned}
& f_{4}(\lambda) e^{2 \lambda^{2}+\lambda^{4} / 9} \leq f_{4}(14 / 25) e^{2(14 / 25)^{2}+(14 / 25)^{4} / 9} \leq \\
& \leq f_{4}(1 / 2) e^{2(14 / 25)^{2}+(14 / 25)^{4} / 9}=0.968649<1, \lambda \in\left[\frac{14}{25}, \frac{99}{100}\right) .
\end{aligned}
$$

Нам осталось доказать неравенство (3) на отрезке $[1,3 / 2)$. В этом случае имеем

$$
\begin{aligned}
& f_{4}(\lambda)=-\frac{3}{16} \lambda^{4}+\frac{5}{8} \lambda^{3}-\frac{3}{16} \lambda^{2}-\frac{37}{32} \lambda+1 ; f_{4}^{\prime}(\lambda)=-\frac{3}{4} \lambda^{3}+\frac{15}{8} \lambda^{2}-\frac{3}{8} \lambda-\frac{37}{32} \\
& f_{4}^{\prime}(\lambda)+\left(4 \lambda+\frac{16}{4 n} \lambda^{3}\right) f_{4}(\lambda)=-\frac{1}{12} \lambda^{7}+\frac{5}{18} \lambda^{6}-\frac{5}{6} \lambda^{5}+\frac{143}{72} \lambda^{4}-\frac{19}{18} \lambda^{3}-\frac{11}{4} \lambda^{2}+\frac{29}{8} \lambda-\frac{37}{32} .
\end{aligned}
$$

Так как функция $f_{4}(\lambda), \lambda \geq 0$, убывает, то

$$
\begin{aligned}
& f_{4}(\lambda) e^{2 \lambda^{2}+\lambda^{4} / 9} \leq f_{4}(53 / 50) e^{2\left(2(54 / 50)^{2}+(54 / 50)^{4} / 9\right.}=0.855641<1 \text { для } 53 / 50 \leq \lambda \leq 54 / 50 ; \\
& f_{4}(\lambda) e^{2 \lambda^{2}+\lambda^{4} / 9} \leq f_{4}(7 / 5) e^{2(29 / 20)^{2}+(29 / 20)^{4} / 9}=0.9255<1 \text { для } 7 / 5 \leq \lambda \leq 29 / 20 ; \\
& f_{4}(\lambda) e^{2 \lambda^{2}+\lambda^{4} / 9} \leq f_{4}(29 / 20) e^{2(3 / 2)^{2}+(3 / 2)^{4} / 9}=0.910697<1 \text { для } 29 / 20 \leq \lambda \leq 3 / 2 .
\end{aligned}
$$


Заметим, что $[1,3 / 2)=[1,53 / 50) \cup[53 / 50,54 / 50) \cup[54 / 50,7 / 5) \cup[7 / 5,29 / 20) \cup$ $\cup[29 / 20,3 / 2)$. Докажем неравенство (3) на отрезке $[1,53 / 50)$. Рассмотрим многочлен $P_{4}(\lambda)=f_{4}^{\prime}(\lambda)+\left(4 \lambda+4 \lambda^{3} / 9\right), \lambda \in \mathbb{R}$, и его производные:

$$
\begin{aligned}
& P_{4}^{(1)}(\lambda)=-\frac{7}{12} \lambda^{6}+\frac{5}{3} \lambda^{5}-\frac{25}{6} \lambda^{4}+\frac{143}{18} \lambda^{3}-\frac{19}{6} \lambda^{2}-\frac{11}{2} \lambda+\frac{29}{8} \\
& P_{4}^{(2)}(\lambda)=-\frac{7}{2} \lambda^{5}+\frac{25}{3} \lambda^{4}-\frac{50}{3} \lambda^{3}+\frac{143}{6} \lambda^{2}-\frac{19}{3} \lambda-\frac{11}{2} \\
& P_{4}^{(3)}(\lambda)=-\frac{35}{2} \lambda^{4}+\frac{100}{3} \lambda^{3}-50 \lambda^{2}+\frac{143}{3} \lambda-\frac{19}{3} \\
& P_{4}^{(4)}(\lambda)=-70 \lambda^{3}+100 \lambda^{2}-100 \lambda+\frac{143}{3} \\
& P_{4}^{(5)}(\lambda)=-210 \lambda^{2}+200 \lambda-100 \\
& P_{4}^{(6)}(\lambda)=-420 \lambda+200 \\
& P_{4}^{(7)}(\lambda)=-420
\end{aligned}
$$

Непосредственные вычисления показывают, что

$$
\begin{array}{lll}
+, P_{4}(1)=\frac{1}{96}>0 ; & +, P_{4}(53 / 50)=\frac{1205119163}{9375000000000}>0 ; \\
-, P_{4}^{(1)}(1)=-\frac{13}{72}<0 ; & -, P_{4}^{(1)}(53 / 50)=-\frac{89167729709}{562500000000}<0 ; \\
+, P_{4}^{(2)}(1)=\frac{1}{6}>0 ; & +, P_{4}^{(2)}(53 / 50)=\frac{1035722147}{1875000000}>0 ; \\
+, P_{4}^{(3)}(1)=\frac{43}{6}>0 ; & +, P_{4}^{(3)}(53 / 50)=\frac{42153899}{7500000}>0 ; \\
-, P_{4}^{(4)}(1)=-\frac{1100417}{3}<0 ; & -, P_{4}^{(4)}(53 / 50)=-\frac{37500}{30989}<0 \\
-, P_{4}^{(5)}(1)=-110<0 ; & -, P_{4}^{(5)}(53 / 50)=-\frac{1250}{5}<0 \\
-, P_{4}^{(6)}(1)=-220<0 ; & -, P_{4}^{(6)}(53 / 50)=-\frac{1226}{5}<0 \\
-, P_{4}^{(7)}(1)=-420<0 ; & -, P_{4}^{(7)}(53 / 50)=-420<0 .
\end{array}
$$

По теореме Будана-Фурье $P_{4}(\lambda)$ не имеет вещественных корней на сегменте $[1,53 / 50]$. Так как $P_{4}(1)=1 / 96>0$, многочлен $P(\lambda)$ положителен на сегменте $[1,53 / 50]$. Из (17) следует, что

$$
f_{4}(\lambda) e^{2 \lambda^{2}+\lambda^{4} / 9} \leq f_{4}(53 / 50) e^{2(53 / 50)^{2}+(53 / 50)^{4} / 9}=0.776938<1 \text { для } 1 \leq \lambda \leq 53 / 50 .
$$

Применим теорему Будана-Фурье для доказательства неравенства $P_{4}(\lambda)<0$ для $27 / 25=54 / 50 \leq \lambda \leq 7 / 5$. Для этого нам потребуются следующие вычисления:

$$
\begin{array}{lll}
-, P_{4}(27 / 25)=-\frac{571111333}{195312500000}<0 ; & -, P_{4}(7 / 5)=-\frac{149057}{22500000}<0 ; \\
-, P_{4}^{(1)}(27 / 25)=-\frac{285915157}{1953125000}<0 ; & +, P_{4}^{(1)}(7 / 5)=\frac{93067}{1125000}>0 ; \\
+, P_{4}^{(2)}(27 / 25)=\frac{12866401}{19531250}>0 ; & -, P_{4}^{(2)}(7 / 5)=-\frac{3697}{18750}<0 ;
\end{array}
$$




$$
\begin{array}{lll}
+, P_{4}^{(3)}(27 / 25)=\frac{2347739}{468750}>0 ; & -, P_{4}^{(3)}(7 / 5)=-\frac{10021}{750}<0 ; \\
-, P_{4}^{(4)}(27 / 25)=-\frac{298811}{9375}<0 ; & -, P_{4}^{(4)}(7 / 5)=-\frac{6631}{75}<0 ; \\
-, P_{4}^{(5)}(27 / 25)=-\frac{16118}{125}<0 ; & -, P_{4}^{(5)}(7 / 5)=-\frac{1158}{5}<0 ; \\
-, P_{4}^{(6)}(27 / 25)=-\frac{1268}{5}<0 ; & -, P_{4}^{(6)}(7 / 5)=-388<0 \\
-, P_{4}^{(7)}(27 / 25)=-420<0 ; & -, P_{4}^{(7)}(7 / 5)=-420<0 .
\end{array}
$$

По теореме Будана-Фурье $P_{4}(\lambda)$ не имеет вещественных корней на сегменте $[27 / 25,7 / 5]$. Т.к. $P_{4}(27 / 25)<0$, многочлен $P_{4}(\lambda)$ отрицателен при $\lambda \in[27 / 25,7 / 5]$. Из (17) следует, что

$$
f_{4}(\lambda) e^{2 \lambda^{2}+\lambda^{4} / 9} \leq f_{4}(27 / 25) e^{2(27 / 25)^{2}+(27 / 25)^{4} / 9}=0.776609<1,27 / 25 \leq \lambda \leq 7 / 5 .
$$

Это завершает доказательство утверждения.

Благодарности. Благодарю своего научного руководителя профессора В.M. Круглова за постановку задачи и полезные советы при работе над статьей.

\section{Заключение}

В статье доказано неравенство

$$
\mathbb{P}\left\{\sqrt{n} \sup _{|x|<\infty}\left(F_{n}(x)-F(x)\right)>\lambda\right\} \leq \exp \left\{-2 \lambda^{2}-\lambda^{4} / 36 n\right\}
$$

для $n \geq 39, \min \left\{\gamma n^{-1 / 6}, \sqrt{\ln 2 / 2}\right\} \leq \lambda \leq \sqrt{n} / 2, \gamma=1.0841$.

Для частных случаев $n=2,3,4$ доказана более сильная оценка

$$
\mathbb{P}\left\{\sqrt{n} \sup _{|x|<\infty}\left(F_{n}(x)-F(x)\right)>\lambda\right\} \leq \exp \left\{-2 \lambda^{2}-4 \lambda^{4} / 9 n\right\} .
$$

\section{Список литературы}

[1] Смирнов Н.В. Приближение законов распределения случайных величин по эмпирическим данным // Успехи математических наук. 1944. № 10. С. 179206.

[2] Dvoretzky A., Kiefer J., Wolfowitz J. Asymptotic minimax character of the sample distribution function and of the classical multinomial estimator // The Annals of Mathematical Statistics. 1956. Vol. 27, № 3. Pp. 642-669.

[3] Massart P. The Tight Constant in the Dvoretzky-Kiefer-Wolfowitz Inequality // The Annals of Probability. 1990. Vol. 18, № 3. Pp. 1269-1283.

[4] Feller W. An Introduction to Probability Theory and Its Applications. New York: Wiley, 1968. 509 p. 


\section{Образец цитирования}

Ташков И.А. Улучшение неравенства Массарта для статистики Смирнова // Вестник ТвГУ. Серия: Прикладная математика. 2018. № 1. С.5-20. https://doi.org/10.26456/vtpmk489

\section{Сведения об авторах}

\section{1. Ташков Иван Андреевич}

студент факультета вычислительной математики и кибернетики Московского государственного университета им. М.В. Ломоносова.

Россия, 119992, г. Москва, ГСП-1, Воробъевы горы, МГУ им. М.В. Ломоносова. E-mail: tashkov95@ya.ru 


\title{
AN IMPROVEMENT OF MASSART'S INEQUALITY FOR THE DISTRIBUTION OF SMIRNOV'S STATISTIC
}

\author{
Tashkov Ivan Andreevich \\ Student of Faculty of Computational Mathematics and Cybernetics, \\ Lomonosov Moscow State University \\ Russia, 119991, Moscow, GSP-1, 1-52, Leninskiye Gory, MSU. \\ E-mail: tashkov95@ya.ru
}

Received 10.12.2017, revised 25.12.2017.

Let $F_{n}$ be the empirical distribution function for a sample of independent identically distributed random variables with distribution function $F$. The main result is the inequality

$$
\mathbb{P}\left\{\sqrt{n} \sup _{|x|<\infty}\left(F_{n}(x)-F(x)\right)>\lambda\right\} \leq \exp \left\{-2 \lambda^{2}-\lambda^{4} / 36 n\right\}
$$

for $n \geq 39, \min \left\{\gamma n^{-1 / 6}, \sqrt{\ln 2 / 2}\right\} \leq \lambda \leq \sqrt{n} / 2, \gamma=1.0841$. It is also proved for the same $n$ and $\lambda \leq \sqrt{n} / 2$ that

$$
\mathbb{P}\left\{\sqrt{n} \sup _{|x|<\infty}\left(F_{n}(x)-F(x)\right)>\lambda\right\} \leq 2 \exp ^{(\ln 2)^{2} /(144 n)} \exp \left\{-2 \lambda^{2}-\lambda^{4} / 36 n\right\} .
$$

In particular cases $n=2,3,4$ it is proved that

$$
\mathbb{P}\left\{\sqrt{n} \sup _{|x|<\infty}\left(F_{n}(x)-F(x)\right)>\lambda\right\} \leq \exp \left\{-2 \lambda^{2}-4 \lambda^{4} / 9 n\right\} .
$$

Keywords: distribution of Smirnov's statistics, exponential inequalities.

\section{Citation}

Tashkov I.A. An improvement of Massart's inequality for the distribution of Smirnov's statistic. Vestnik TvGU. Seriya: Prikladnaya Matematika [Herald of Tver State University. Series: Applied Mathematics], 2018, no. 1, pp. 5-20. (in Russian). https://doi.org/10.26456/vtpmk489

\section{References}

[1] Smirnov N.V. Approximation of the laws of distribution of random variables from empirical data. Uspekhi Matematicheskikh Nauk [Successes of Mathematical Sciences], 1944, no. 10, pp. 179-206. (in Russian)

[2] Dvoretzky A., Kiefer J., Wolfowitz J. Asymptotic minimax character of the sample distribution function and of the classical multinomial estimator. The Annals of Mathematical Statistics, 1956, vol. 27(3), pp. 642-669.

[3] Massart P. The Tight Constant in the Dvoretzky-Kiefer-Wolfowitz Inequality. The Annals of Probability, 1990, vol. 18(3), pp. 1269-1283.

[4] Feller W. An Introduction to Probability Theory and Its Applications. Wiley, New York, 1968. 509 p. 